Postprint version. Please refer to the publisher's version as follows:

Zapounidou S., Sfakakis M., Papatheodorou C. (2013) Highlights of Library Data Models in the Era of Linked Open Data. In: Garoufallou E., Greenberg J. (eds) Metadata and Semantics Research. MTSR 2013. Communications in Computer and Information Science, vol 390. Springer, Cham doi: 10.1007/978-3-319-03437-9 38

The final publication is available at link.springer.com

\title{
Highlights of library data models in the era of Linked Open Data
}

\author{
Sofia Zapounidou ${ }^{1}$, Michalis Sfakakis ${ }^{1}$, Christos Papatheodorou ${ }^{1,2}$ \\ ${ }^{1}$ Department of Archives and Library Science, Ionian University, Corfu, Greece \\ ${ }^{2}$ Digital Curation Unit, IMIS, “Athena” Research Center, Athens, Greece \\ \{112zapo, sfakakis, papatheodor\}@ionio.gr
}

\begin{abstract}
Semantic Web technologies and Linked data form a new reference framework for libraries. The library community aims to integrate its data with the Semantic Web and as a result new library data models have been developed. In this context, significant research effort focuses on the alignment between the library models with relevant models developed by other communities in the cultural heritage domain. However there exist several issues concerning the interoperability between all these data models. This paper seeks to contribute in the interoperability of four models, namely FRBR, FRBRoo, EDM and BIBFRAME. It highlights the commonalities and the divergences between them by using a case bibliographic record and by exploring how this record is represented by each one of them.
\end{abstract}

Keywords: semantic web, library data models, linked data, FRBR, FRBRoo, EDM, BIBFRAME

\section{Introduction}

Libraries develop and preserve rich metadata for their holdings. Exchange of these metadata between libraries is made through specific bibliographic standards. The most well known is the MARC (MAchine Readable Cataloguing) data structure standard [1], developed in the 1960s and widely used since then by libraries world- 
wide. Despite its success and its many updates since its first development, MARC faces the challenges to enable provision and use of library data by third party applications, as well as to adapt its framework to the current trends of the metadata models [2].

The insufficiencies of current bibliographic data representation standards have been well studied by experts and library-related international organizations [3-8]. As a result, there is a growing interest in establishing meaningful representation and communication of bibliographic data in the environment of the web of data, which has already appeared exploiting Semantic Web technologies that enable the publication and consumption of structured data. These technologies known as Linked Data [9] provide a new reference framework for libraries aspiring to integrate their data into the Semantic Web [2], thus providing very rich datasets that may be used, exploited and extended by other libraries or communities. Some pilot efforts have already been undertaken by the library community to redefine the bibliographic universe in accordance with the new reference framework described above. These different interpretations are expressed almost simultaneously and may cause interoperability problems, making integration of library data into the Semantic Web not a simple task. Library institutions and stakeholders, library data, and library processes need to be defined explicitly according to the requirements and benefits of the new data publishing framework [2].

The aim of this paper is to contribute in the interoperability of such approaches. Therefore, it tries to compare bibliographic data models developed by different initiatives in the library community with the aim of discovering the common ground between them. For this purpose four data models are explored: FRBR, FRBRoo, Europeana Data Model (EDM) and BIBFRAME model. Since libraries mainly collect monographs, this paper examines the way monographs could be conceptually modeled by the mentioned models. The monograph chosen as a case study to be investigated is a well-known literary work, 'Don Quixote de la Mancha'. It consists of two separate works: the first one was published in 1605 with the title 'El ingenioso hidalgo don Quixote de la Mancha' and the second one was published in 1615 with the title 'Segunda parte del ingenioso cauallero don Quixote de la Mancha'. The monograph selected is an English translation of both parts incorporated in one single volume. The description of the monograph was found in the bima0000074081MARC21 record downloaded as a MARCXML file from the National Library of Spain Catalog. For the mentioned models and the given monograph the paper investigates the following research questions:

- Is there any consensus on their conceptual modeling view in the case of monographs?

- Can the different point of views converge and how?

- Can bibliographic data following such models, be integrated?

The next section of the paper provides a short description of the followed approach. A short description of the mentioned data models along with a brief representation of the monograph chosen is provided in section 3 . In section 4 a comparison is presented aiming to highlight the main characteristics of each model and its expressiveness in 
revealing the semantics of the information provided by the bibliographic records that share similar characteristics with the monograph in hand. Finally, the lessons learnt are summarized in the conclusions section.

\section{Overview of the approach}

In order to highlight the commonalities and divergences at conceptual level between the used models, it was decided at the beginning of this investigation to use a real representation of a well known monograph. Therefore, the 'Don Quixote de la Mancha' bibliographic record was selected as an appropriate case to start representing the most fundamental aspects of the models, while it could be utilized for more complicated representations by the examined models. This record was selected because it represents a multipart monograph published in a single volume. Additionally, the parts of this work have also been published as independent volumes. Therefore, this case provides the ability to build more complex instances of the models and to assess their expressiveness. Moreover, this record was used as a case study from a Europeana Task Force group [10] and therefore we can verify its core representations for the FRBR and FRBRoo models, while for the representation of the BIBFRAME model we used the representation produced from the bibframe.org [11] tools.

Table 1 presents a labeled version of the MARC record containing the most significant metadata of the monograph required to populate the most characteristic and respective classes of the models. For this purpose, all the mentioned models were studied in terms of elucidating the semantics of their classes and relationships. Then, the classes of each of them that can model the 'Don Quixote de la Mancha' record were identified and a graphical representation for each model for the monograph on hand was sketched. These graphical representations accompany the models' descriptions found in the next section. All graphical representations were afterwards compared to reveal both common ground and differentiations regarding the conceptual modeling approaches adopted by the models under investigation. For readability purposes, it should be stated that the graphical representations are simplified and thus some straightforward entities were deliberately omitted, such as authors, contributors etc as well as complete URIs required by the RDF language.

Table 1. Labeled version of the bima0000074081 MARCXML record

\begin{tabular}{|l|l|}
\hline Personal name / Author & \multicolumn{1}{c|}{ Cervantes Saavedra, Miguel de (1547-1616) } \\
\hline Uniform title & {$[$ Don Quijote de la Mancha. Inglés] } \\
\hline Title & $\begin{array}{l}\text { The history of the most renowned Don Quixote of Mancha } \\
\text { [Texto impreso]: and his trusty squire Sancho Pancha [sic] } / \\
\text { now made English according to the humour of our modern } \\
\text { language and adorned with seueral copper plates by J.P. }\end{array}$ \\
\hline Publisher/Date & $\begin{array}{l}\text { London : printed by Thomas Hodgkin and sold by William } \\
\text { Whitwood ..., 1687 }\end{array}$ \\
\hline
\end{tabular}




\begin{tabular}{|l|l|}
\hline Physical description & {$[16], 616,[3]$ p., [7] h. de lám.; Fol. } \\
\hline Contents & Partes primera y segunda \\
\hline Title note & $\begin{array}{l}\text { Las iniciales J.P. corresponden al traductor, J. Philips, co- } \\
\text { mo consta en la dedicatoria }\end{array}$ \\
\hline Added author & $\begin{array}{l}\text { Hodgkin, Thomas (s. XVII), imp.; Whitwood, William, ed.; } \\
\text { Phillips, John (1631-1706), tr. }\end{array}$ \\
\hline
\end{tabular}

\section{Library Data Models: representation of the case study}

\subsection{Functional Requirements for Bibliographic Records -(FRBR)}

Functional Requirements for Bibliographic Records [6] is the result of a long-term study initiated by one of the resolutions adopted by the 1990 Stockholm Seminar on Bibliographic Records. That resolution asked for a clear delineation of "the functions performed by the bibliographic record with respect to various media, various applications, and various user needs" [12].

The FRBR study presented a conceptual entity-relationship model that defined key bibliographic entities, their attributes and the relationships between them. The bibliographic entities were grouped into three groups: Group 1 entities refer to intellectual or artistic products (Work, Expression, Manifestation, Item), Group 2 entities refer to people (Person) and/or corporate bodies (Corporate Body) that create, publish or preserve the Group 1 entities, Group 3 entities are used as topics in the Group 1 entities (Concept, Object, Event, and Place).

In Group1 entities (Work, Expression, Manifestation, Item, also known as WEMI) are defined from the abstract to the concrete level. Work and Expression reflect the content, while Manifestation and Item reflect the physical form of the content. Work refers to the intellectual or artistic creation, Expression refers to the realization of the intellectual or artistic creation, Manifestation refers to the physical embodiment of an expression of a work, Item refers to a single copy of a given manifestation.

Each entity is defined by a set of attributes. For instance a Manifestation is described by 38 attributes, such as title of the manifestation, statement of responsibility, edition/issue designation, place of publication /distribution, date of publication/distribution, fabricator/manufacturer, etc. It should be noticed that most descriptions in library catalogues correspond to the Manifestation entities of the model, while library holdings correspond to Item entities.

The entities of the model are inter-linked by a set of relationships. The entities Work and Expression are correlated with the relationship is realized through. Moreover, the relationship is created correlates a Work with the entities of Group 2 (Person, Corporate Body). The entities Expression and Manifestation are interlinked though the relationship is embodied in, while the entities Manifestation and Item are correlated through the relationship is exemplified by. Expression is correlated with the entities 
of Group 2 through the relationship is realized by, while Manifestation and Item are correlated with the same group of entities via the relationships is produced and is owned by respectively. Furthermore, the entities of Group 3 are correlated with the entities of the Groups 1 and 2 through the relationship has as subject.

Even though the FRBR study defined the entities in each group, it focused on Group 1 entities. The entities in the other two groups involved authority data and were further analyzed in two subsequent reports, namely Functional Requirements for Authority Data - FRAD [13] and Functional Requirements for Subject Authority Data - FRSAD [14]. All these models are also known as the FRBR family of models. Gordon Dunsire expressed the FRBR family of models in RDF and made these RDF vocabularies available through the Open Metadata Registry (http://metadataregistry.org/).

Figure 1 depicts briefly the instances of the four entities derived from the MARC21 record in question: the instance of the Work is 'Don Quixote de la Mancha', its Expression in an English translation by J.Philips, a Manifestation of the English translation published in 1687 by William Whitwood and printed by Thomas Hodgkin and an Item of this Manifestation, which in this case is a digitized copy.

\begin{tabular}{|c|c|}
\hline frbr:Work & \multirow[b]{3}{*}{$\begin{array}{l}\text { Pancha [sic] / now made English according ... by J.P." } \\
\text { frbr:isProducerPersonOf= Hodgkin, Thomas } \\
\text { frbr:isProducerPersonOf= William Whitwood }\end{array}$} \\
\hline "Don Quixote de la Mancha" & \\
\hline $\begin{array}{l}\text { is realized through } \\
\qquad \quad \text { is embodied in }\end{array}$ & \\
\hline frbr:Expression & $\downarrow$ is exemplified by \\
\hline "The history of the most & frbr:Item \\
\hline $\begin{array}{l}\text { renowned Don Quixote of } \\
\text { Mancha" / eng by John Phillips }\end{array}$ & $\begin{array}{l}\text { http://bibliotecadigitalhispanica.bne.es:80/webclient/Delivery } \\
\text { Manager?pid=1811612\&amp;custom_att_2=simple_viewer }\end{array}$ \\
\hline
\end{tabular}

Figure 1. A monograph represented by FRBR

\subsection{Functional Requirements for Bibliographic Records object-oriented version (FRBRoo)}

The object-oriented version of FRBR, known as FRBRoo, is a harmonization of the CIDOC-CRM and the FRBR models. CIDOC CRM [15] is a reference model which represents conceptually the cultural heritage domain and consists of a hierarchy of 86 entities (or classes) and 137 properties. It ensures semantic integration between different cultural metadata schemas and aims to eliminate their possible semantic heterogeneities. The aim of the harmonization of the two models was the development of an ontology that would enable the representation of bibliographic information, thus facilitating "the integration, mediation, and interchange of bibliographic and museum information" [16]. To address this aim, FRBRoo includes concepts from both FRBR family of models and CIDOC-CRM: FRBR Group 1 entities were further analyzed and refined; temporal entities, events and time processes were included; creation and production processes were also modeled. 
As far the Group 1 entities are concerned, various interpretations of Work have been incorporated into FRBRoo, e.g. F14 Individual Work, F19 Publication Work, F16 Container Work that may respectively correspond to one single work, a work prepared for publication with the publisher's contributions, and an aggregate work. Dimensions of time and place were also expressed in the model to support the description of intellectual creation and physical production processes.

FRBRoo may support uniformly static or dynamic views of the bibliographic universe. It may support the description of bibliographic products independently from their creation, modification and publication processes just as the FRBR family of models does. It also enables more dynamic descriptions by taking into account the dimension of time, and by modeling both creation and production processes and their intellectual or artistic products. FRBRoo results into being a dynamic flexible model that provides cataloguing agencies the means to describe their holdings according to their cataloguing policies, to their view (static or dynamic may it be) of their collections.

Figure 2 provides a representation of the monograph in hand according to FRBRoo. Five classes are utilized for this purpose. The class F15 Complex Work having as instance the 'Don Quixote de la Mancha', the class F22 Self-Contained Expression having as instance the English translation by John Phillips, the class F24 Publication Expression that incorporates the printer's and the publisher's information, the class F3 Manifestation Product Type that carries the title of the F24 Publication Expression as appeared in the title page. Finally, the class F5 Item has as instance a URI to the digitized copy of the monograph.

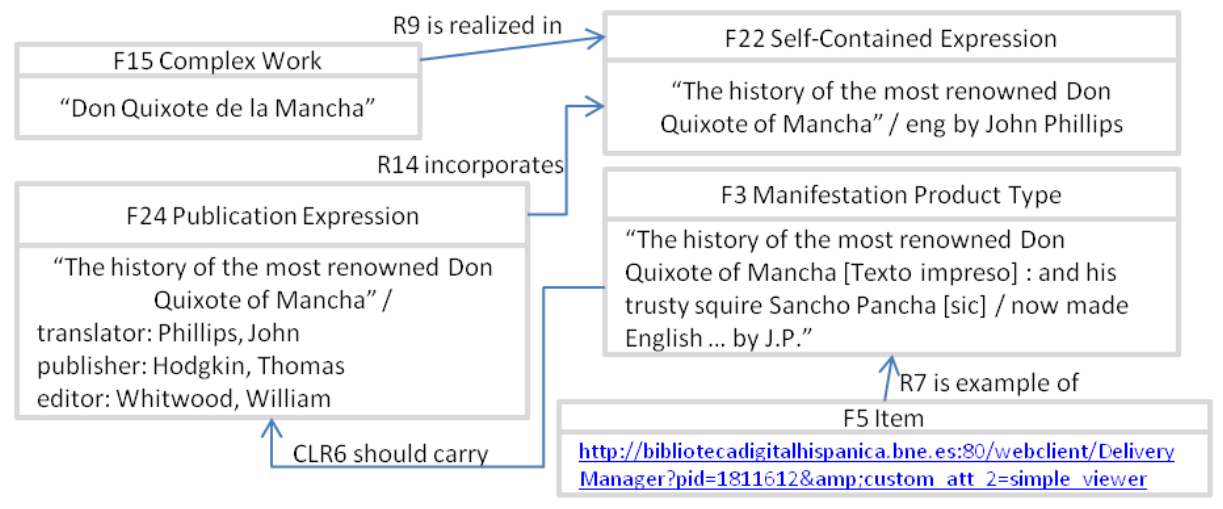

Figure 2. A monograph represented by FRBRoo

\subsection{Europeana Data Model (EDM)}

Europeana (http://www.europeana.eu/) is an aggregation portal providing access to born-digital or digitized cultural heritage content provided by memory institutions all over Europe. Descriptions over Europeana are made with a basic data model called Europeana Semantic Elements [17], which uses 12 elements in addition to the 15 elements of the Dublin Core Metadata Element Set. The Europeana Data Model 
(EDM) has been created to offer an advanced data model that may better express the semantics of the cultural heritage descriptions provided by different communities. No community - driven standard was used as a basis for its development and the Semantic Web framework was taken into account [18].

For each provider, EDM distinguishes between real provided cultural heritage objects (e.g. painting), their digital representations (e.g. digital image of painting), and their descriptions (e.g. metadata about painting). EDM has three core classes, namely edm:ProvidedCHO (for provided Cultural Heritage Object), edm:WebResource (for the edm:ProvidedCHO digital representations) and ore:Aggregation (for the aggregation of the activities made by the provider of the edm:ProvidedCHO).

Alignment of EDM with library metadata is a work in progress. In 2012, two reports have been published $[19,20]$, both taking into consideration the FRBR model, seeking to express FRBR entities with EDM classes, and focusing on specific library materials (monographs, multi-volume works and serials). The latest report was the revised D5.1. alignment report [20] which suggested that all FRBR Group 1 entities (Work, Expression, Manifestation, Item) will be expressed by the edm:ProvidedCHO class and "the distinction between them will lie only in the metadata used and in the relationships expressed"[20]. In this report, the need of further investigation was recognized, suggesting at the same time the integration of FRBRoo entities in EDM using FRBRoo terms.

As a result the EDM - FRBRoo Application Profile Task Force was launched in July 2012. The scope of this taskforce was to build upon the existing mapping of FRBRoo and the EDM provided by the CIDOC CRM [15] by "extending, correcting or restricting it and providing examples for the use of the combined EDM and FRBRoo name-space clusters" [10].The task force ended its work on 30 April 2013.

The Task Force worked with real datasets and sets of research questions for three types of library material: monographs (Don Quixote test case), theatrical plays (Shakespeare's Hamlet) and musical works (1st Symphony by Johannes Brahms and The Rite of Spring by Igor Stravinsky). These datasets were expressed in FRBRoo to enable the selection of basic FRBRoo classes and properties conforming to a set of stated principles. Then a mapping process was carried out to map all the selected FRBRoo classes and properties to EDM. The study resulted in the development of an FRBRoo - EDM application profile. The application profile includes two sets: the EDM classes and properties that may be used in the mapping process directly and the FRBRoo concepts that may be considered as specializations of EDM classes and properties.

Figure 3 presents the representation of the Don Quixote monograph as implemented by EDM, while Figure 4 provides the representation of the monograph as implemented by the EDM - FRBRoo Application Profile. 
edm:providedCHO

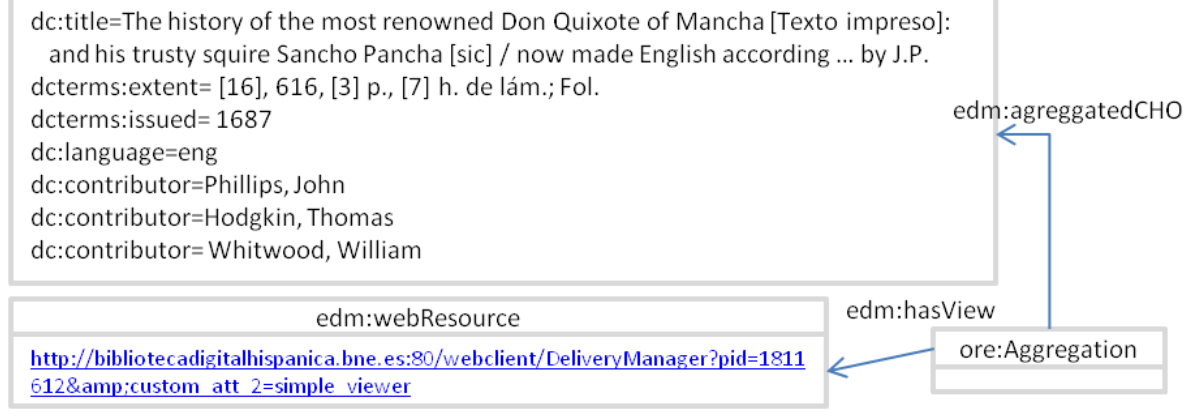

\begin{tabular}{|l|l|l|}
\hline \multicolumn{1}{|c|}{ edm:webResource } & edm:hasView \\
\hline$\underline{\text { http://bibliotecadigitalhispanica.bne.es:80/webclient/DeliveryManager?pid=1811 }}$ \\
\hline$\underline{612 \text { \&amp;custom att 2=simple viewer }}$
\end{tabular}

Figure 3. A monograph represented by EDM

As mentioned the Europeana Data Model holds data at the manifestation level of FRBR. Thus, the monograph in hand is represented by two entities: the edm:provided $\mathrm{CHO}$ that holds all information about the monograph entitled 'The history of the most renowned Don Quixote of Mancha' and published in 1687 by William Whitwood. The other entity is the edm:webResource that links to the digitized copy of the monograph.

The EDM - FRBRoo application profile within the scope of "allow(ing) a better representation of the FRBR Group 1 entities" [21] maps EDM classes to FRBRoo (Figure 4). Thus, the monograph in question is modeled as follows: an instance of the class edm:InformationResource represents the instance of the FRBR Work 'Don Quixote de la Mancha'. A second instance of the class edm:InformationResource is mapped to the FRBR Expression class. The class edm:providedCHO is mapped to the class F24 Publication Expression of FRBRoo and, finally, the class edm:webResource represents the digital copy, which actually carries the information from the publication expression.

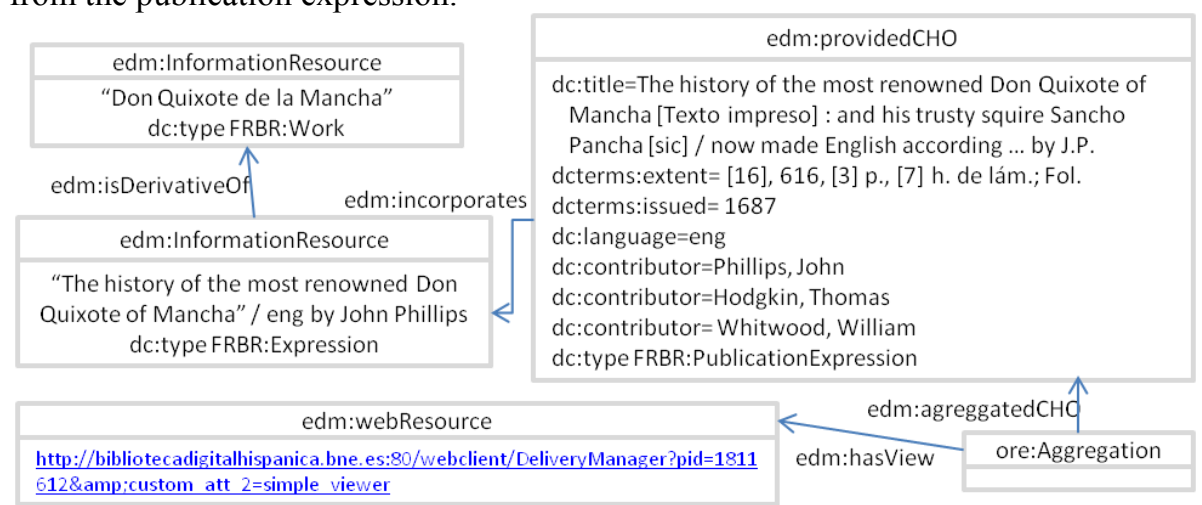

Figure 4. A monograph represented by EDM - FRBRoo 


\subsection{Bibliographic Framework Initiative Data Model (BIBFRAME model)}

In October 2011, Library of Congress announced the launch of a new programme regarding the creation of a new Bibliographic Framework. This framework aims to accommodate many bibliographic formats and data models and the only prerequisite stated is the use of Linked Data technologies and the Resource Description Framework [22]. On May 22, 2012 the Library of Congress announced its cooperation with Eric Miller's Zepheira Group for the development of a bibliographic data model [23]. The draft model was presented on November 2012 and a BIBFRAME mailing list was created for the exchange of ideas and further development of the model.

The BIBFRAME model main classes are: Creative Work, Instance, Authority and Annotation [24]. The class Creative Work reflects the "conceptual essence of the cataloguing item" [24] and seems to be semantically closer to the FRBR Work and Expression entities. The class Instance reflects "an individual, material embodiment of the Work" and seems to be alike to the FRBR Manifestation entity. The class Authority is rather straight-forward; this class is used to identify People, Places, and Organizations involved in the creation or publication of a Work. For the expression of topics, BIBFRAME Authority simply works as a linking mechanism to LC Subject Headings published as linked data at the ID.LOC.GOV site [25]. The class Annotation expresses comments made about a BIBFRAME Work, Instance, Authority. Examples of BIBFRAME annotations are: library holdings, cover arts, sample texts, reviews, etc. The BIBFRAME model is still under development.

The MARC 21 record of the Don Quixote monograph (bima0000074081.xml file) was submitted to the transformation service available at the official BIBFRAME.org website [11]. The transformation service derived two instances of $b f:$ Work and one $b f:$ Instance. The first Work is 'Don Quixote de la Mancha', the second Work is 'The history of the most renowned Don Quixote of Mancha' and its instance published in 1687. The relationship between the second Work and its instance are depicted briefly in Figure 5. Note that the BIBFRAME transformation service did not relate the $b f:$ Work 'Don Quixote de la Mancha' to the $b f:$ Work 'The history of the most renowned Don Quixote of Mancha' even though they were derived from the same MARC record.

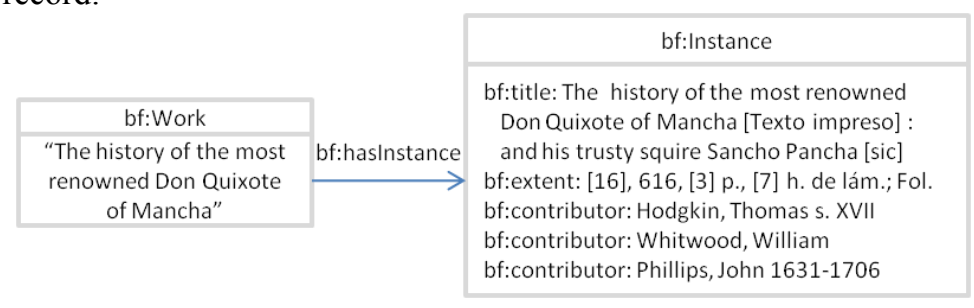

Figure 5. A monograph represented by BIBFRAME 


\section{Discussion}

Modeling the English translation of the 'Don Quixote de la Mancha' under each model's semantics revealed some common ground and some significant differences as well. All models recognize that there is a given number of entities (classes), and that each of them is associated with specific characteristics (attributes). Furthermore, the classes are correlated through a set of relationships that enrich the semantics of each model. Moreover, they all have been somehow expressed in a Semantic Web framework with the re-use of existing or development of new element sets and vocabularies. FRBR has been expressed in RDF by Gordon Dunsire, FRBRoo, EDM and BIBIFRAME are all developed in RDF. Thus, the exact semantics of each model's entity and the semantic differences between them may be identified in detail.

All models make a distinction between content and its carrier. The abstraction levels firstly defined in the FRBR model are recognized by the other three models (FRBRoo, EDM, BIBFRAME) as an important asset for the library community that must be taken into consideration. FRBRoo and EDM associate their entities to the FRBR Group 1 entities. BIBFRAME aims to accommodate FRBR Group 1 entities in the near future. Additionally, there seems to be more common ground between FRBR and FRBRoo models and between EDM and BIBFRAME models. FRBR and FRBRoo models are similar to each other following the basic FRBR four levels of abstraction (Work, Expression, Manifestation, Item). EDM and BIBFRAME are more similar to each other adopting a two-level approach: edm:providedCHO and edm:webResource in EDM, Creative Work and Instance in BIBFRAME.

A main difference is that each model recognizes a different number of abstraction layers. FRBR and FRBRoo model intellectual or artistic creations and their physical embodiments with four classes (also known as WEMI hierarchy), while EDM and BIBFRAME use only two levels. FRBR and FRBRoo go beyond existing bibliographic standards, while the current Europeana Data Model and the BIBFRAME model seem to build upon the semantics of MARC21. In EDM all information concerning WEMI are added to the edm:providedCHO [20] and BIBFRAME has been initiated as an "... effort to translate MARC 21 to a Linked Data model" [24].

Another difference is that information about contributors, namely translator J.Phillips, publisher W. Whitwood and printer T. Hodgkin, is represented in different entities in each model. In FRBR and FRBRoo the translator's contribution determines the creation of an frbr:Expression, while in EDM and BIBFRAME model the translator's contribution is described in a lower level of each model's hierarchy: in edm:provided $\mathrm{CHO}$ and bf:Instance respectively. FRBR includes the information about the printer and the publisher in frbr:Manifestation, while FRBRoo contextualizes them through a new class, F24 Publication Expression. In the other two models the semantics of these pieces of information are not discriminated and are given in edm:provided $\mathrm{CHO}$ and $b f:$ Instance respectively.

These differences reflect the models' disparate creation contexts and the different modeling approach they follow. FRBR was created to express intellectual creations in a library setting. It was developed according to the entity-relationship model and its "Group 1 entities represent the different aspects of user interests in the products of 
intellectual or artistic endeavour"[12]. As a result, four levels have been identified to enable search, identification, selection, and obtaining library materials. A user, for example, may search for a work entitled 'Don Quixote de la Mancha' (Work), search this Work only in its English versions (Expression), search a specific publication where contributions of interest to the user have been made (Manifestation) and then obtain a physical or digital copy (Item). FRBRoo follows the CIDOC CRM logic which describes the history of objects over time and results in capturing the history of a library object starting from its conception and ending with its last ownership status. FRBRoo extends FRBR following an object-oriented approach and includes CIDOC CRM entities. The aim was to "model processes starting from initial idea to the item a user holds" [16]. The FRBR Group 1 entities were further analyzed and more classes that qualify Works, Expressions, Manifestations were identified.

EDM has been developed as a simple data model that providers can easily use to make their digitized resources available through the Europeana portal. It is a generic model that "adopts an open, cross-domain Semantic Web-based framework" [18] and may uniformly accommodate standards from the libraries, archives and museums communities. Its two levels of abstraction, edm:providedCHO and edm:webResource are mapped to the FRBR Manifestation and Item entities respectively. The recently developed EDM-FRBRoo mapping proved that only a subset of the semantically rich FRBRoo classes is needed to describe the Europeana content. As a result the new application profile is closer to FRBR and FRBRoo models [10].

BIBFRAME is a linked data model developed with the aim of providing a simplified model to "which various content models can be mapped" [24] and at the present initial stage focuses on integrating data from MARC21 and RDA records into the Semantic Web. Regarding its dual approach and the relationship with FRBR, it is stated that "the origin of the Work/Instance aspects of the BIBFRAME can reflect the FRBR relationships in terms of a graph rather than as hierarchical relationships, after applying a reductionist technique" [24].

\section{Conclusions}

This first investigation revealed common ground and divergences among the four tested models. The comparison of the FRBR, FRBRoo, EDM and BIBFRAME models performed on the MARC21 record 'Don Quixote de la Mancha' has revealed that interoperability is not an impossible task to undertake especially when certain levels of interoperability are considered. As far as the technical aspect of interoperability is considered, it could be concluded that it is achievable because all models are developed in RDF. Semantic interoperability is not a straightforward issue but it has been shown that there is a consensus regarding conceptual modeling as far as the distinction of content and its carrier is concerned.

Each model has its own classes and properties defining its own semantics. Thus, it may be assumed that a mapping between the models may be possible. The successful EDM-FRBRoo mapping advocates in favor of this assumption. Taken into consideration that the EDM-FRBRoo mapping was accomplished in a very specific context, 
that of Europeana, any mapping between the four models should be made with a specific context in mind. This specific context is a matter of achieving organizational interoperability in the library domain. Consensus among the library community regarding the representation of collections and materials may and should be built. This is the prerequisite for developing application profiles and cataloguing guidelines that could be usable and implementable in new advanced library systems.

Further investigations should be performed using a larger set of bibliographic records that comprise more cases of bibliographic descriptions. Then, considerably evaluation results will improve the quality of the mappings between the data models and contribute in the semantic interoperability between them.

\section{References}

1. Library of Congress: MARC Standards, http://www.loc.gov/marc/

2. W3C Library Linked Data Incubator Group: Library Linked Data Incubator Group Final Report: W3C Incubator Group Report 25 October 2011. Report, W3C (2011).

3. Heaney, M.: Object-oriented cataloging. Information Technology and Libraries. 14, 135 (1995).

4. Ridley, M.: Beyond MARC. In: Weihs, J. (ed.) International Conference on the Principles and Future Development of AACR Toronto, Canada, October 23-25, 1997. Canadian Library Association; Library Association Publishing; American Library Association, Ottawa; London; Chicago (1998).

5. Howarth, L.C.: Content versus Carrier. In: Weihs, J. (ed.) International Conference on the Principles and Future Development of AACR Toronto, Canada, October 23-25, 1997. pp. 148-157. Canadian Library Association; Library Association Publishing; American Library Association, Ottawa; London; Chicago (1998).

6. IFLA Study Group on the Functional Requirements for Bibliographic Records: Functional Requirements for Bibliographic Records Final Report. Saur, München (1998).

7. Tennant, R.: A Bibliographic Metadata Infrastructure for the 21st Century. Library Hi Tech. 22, 175-181 (2004).

8. Calhoun, K.: The changing nature of the catalog and its integration with other discovery tools. Report, Library of Congress (2006).

9. W3C: Linked data, http://www.w3.org/standards/semanticweb/data.

10. Task Force EDM FRBRoo, http://pro.europeana.eu/web/network/europeana-tech//wiki/Main/Task+Force+EDM+FRBRoo.

11. Library of Congress, Zepheira Inc.: BIBFRAME.org, http://bibframe.org/.

12. IFLA Study Group on the Functional Requirements for Bibliographic Records: Functional Requirements for Bibliographic Records. Final Report, IFLA (2009).

13. International Federation of Library Associations and Institutions: Functional Requirements for Authority Data: A conceptual model. K.G.Saur, München (2009).

14. IFLA Working Group on the Functional Requirements for Subject Authority Records: Functional Requirements for Subject Authority Data (FRSAD): A Conceptual Model, Report, IFLA (2010).

15. Bœuf, P. Le, Doerr, M., Ore, C.E., Stead, S.: Definition of the CIDOC Conceptual Reference Model, version 5.1. (draft). ICOM/CIDOC CRM Special Interest Group (2013). 
16. Bekiari, C., Doerr, M., Bœuf, P. Le, Riva, P.: FRBR object-oriented definition and mapping from FRBRER, FRAD and FRSAD (version 2.0). International Working Group on FRBR and CIDOC CRM Harmonisation (2013).

17. Europeana Project: Europeana Semantic Elements Specification Version 3.4.1. Europeana (2012).

18. Isaac, A.: Europeana data model primer. Europeana Project (2011).

19. Europeana Project: Definition of the Europeana Data Model elements Version 5.2.3. Europeana Project (2012).

20. Angjeli, A., Bayerische, M., Chambers, S., Charles, V., Clayphan, R., Deliot, C., Eriksson, J., Freire, N., Huber, A., Jahnke, A., Pedrosa, G., Phillips, V., Pollecutt, N., Robson, G., Seidler, W., Rühle, S.: D5.1 Report on the alignment of library metadata with the European Data Model (EDM) Version 2.0. Report, Europeana Project (2012).

21. Doerr, M., Gradmann, S., Leboeuf, P., Aalberg, T., Bailly, R., Olensky, M.: Final Report on EDM - FRBRoo Application Profile Task Force. Report, Europeana Project (2013).

22. Library of Congress: A Bibliographic Framework for the Digital Age. Report, Library of Congress (2011).

23. Library of Congress: The Library of Congress Announces Modeling Initiative (05/22/12), http://www.loc.gov/bibframe/news/bibframe-052212.html.

24. Miller, E., Ogbuji, U., Mueller, V., MacDougall, K.: Bibliographic Framework as a Web of Data: Linked Data Model and Supporting Services. Report, Library of Congress (2012).

25. BIBFRAME.org: On BIBFRAME Authority 22 May 2013, http://bibframe.org/documentation/bibframe-authority/. 